\title{
Thermal Buckling Behaviors of FGM under Aerodynamic Flows
}

\author{
Chang-Yull Lee, Taegyu Kim and Henzeh Leehim
}

Department of Aerospace Engineering, Chosun University, 309 Pilmun-daero, Dong-gu, Gwangju, South Korea

\begin{abstract}
Post-buckling behaviors of the functionally graded material (FGM) panel under thermal and aerodynamic loading are studied. The material properties of the model vary continuously from one interface to the other in the thickness direction according to distributions in terms of the volume fractions. The two types of material such as power-law FGM (P-FGM) and sigmoid FGM (S-FGM) are compared according to the volume fractions throughout the thickness direction. Newton-Raphson iterative method applied for solving the nonlinear equations of the thermal post-buckling analysis.
\end{abstract}

Keywords: P-FGM, S-FGM, Third-order piston theory

\section{Introduction}

The composite materials are developed for various engineering fields due to the light-weight, excellent strength characteristics et al. However, delamination may occur at the interface of the two materials due to the mismatch of the properties. Especially, the composite may appear remarkably cracking or de-bonding in a high temperature environment.

Therefore, the concept of functionally graded materials (FGMs) has been introduced [1] to overcome these drawbacks in composite materials. The materials have been generated microscopically inhomogeneous composites made from a mixture of ceramics and metals. Up to now, many kinds of research works have been studied on thermal stress and buckling analyses of the FG plates [2]. Especially, Power-law functions is commonly used to describe the variations of material properties of FGM. However, the stress concentrations occur in one of the interfaces from the material is continuously but rapidly changing. Therefore, Chung and Chi [3] proposed a sigmoid FGM, which was composed of two power-law functions to define a new volume fraction.

Recently, a large number of high speed vehicles has been developed, thus the hypersonic flow has been widely studied in many fields. A few researchers have studied flight vehicles in the hypersonic regime, and the unsteady nonlinear piston theory is more suitable for the analysis of the hypersonic phenomena [4].

FGM plate models have been studied widely, therefore the post-buckling behaviors of P-FGM and S-FGM under thermal and aerodynamic loading are investigated in this work.

\section{Material Properties}

FGM is generally made of ceramic and metal. Material properties distribution are two main functions such as P-FGM and S-FGM are employed for analysis of FGM structures.

P-FGM model is defined as:

$$
V_{c}(z)=\left(\frac{z}{h}+\frac{1}{2}\right)^{k} \quad(0 \leq k<\infty), \quad V_{c}+V_{m}=1
$$

where $V$, the superscript $k$, the subscript $c$ and $m$ represent the volume fraction, the volume fraction index, ceramic and metal, respectively.

S-FGM is proposed to ensure smooth distribution of stresses among all the interfaces by using two powerlaw functions [3]. 


$$
\begin{gathered}
V_{c 1}(z)=1-\frac{1}{2}\left(1-\frac{2 z}{h}\right)^{k} \quad(0 \leq z \leq h / 2), \\
V_{c 2}(z)=\frac{1}{2}\left(1+\frac{2 z}{h}\right)^{k} \quad(-h / 2 \leq z \leq 0) \\
V_{c}+V_{m}=1
\end{gathered}
$$

Figure 1 (a)-(b) show the distribution of volume fraction $V c$ through the thickness of the model for various values of the volume fraction index.
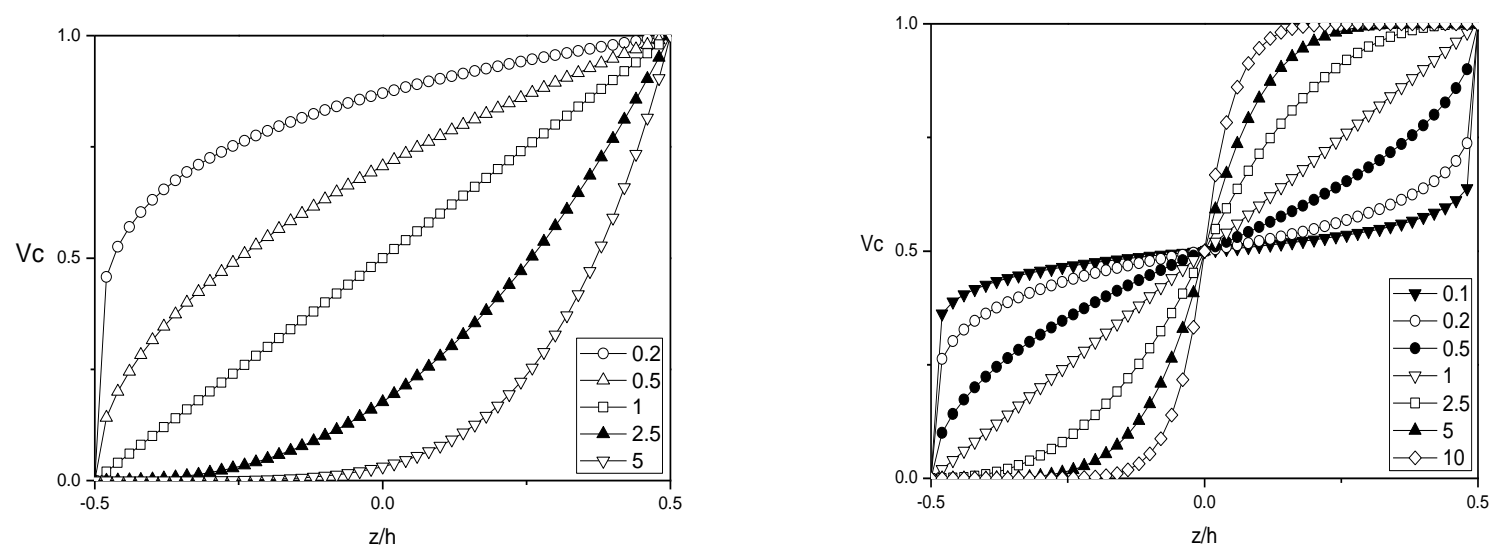

Fig. 1: Volume fraction distributions. (a) P-FGM, (b) S-FGM

\section{Formulations}

Governing equations are derived by the principle of virtual work and numerical solutions are obtained by using the finite element method.

\subsection{Constitutive equations}

Strains based on the First-order Shear Deformation theory (FSDT) are represented as:

The constitutive equations can be written as:

$$
\begin{aligned}
& \left\{\begin{array}{l}
\mathbf{N} \\
\mathbf{M}
\end{array}\right\}=\left[\begin{array}{ll}
\mathbf{A} & \mathbf{B} \\
\mathbf{B} & \mathbf{D}
\end{array}\right]\left\{\begin{array}{l}
\boldsymbol{\varepsilon} \\
\boldsymbol{\kappa}
\end{array}\right\}-\left\{\begin{array}{l}
\mathbf{N}_{\Delta \mathbf{T}} \\
\mathbf{M}_{\Delta \mathbf{T}}
\end{array}\right\} \\
& \mathbf{Q}=\mathbf{A}_{\mathrm{s}} \boldsymbol{\gamma}
\end{aligned}
$$

where $\mathrm{N}, \mathrm{M}, \mathrm{Q}, \mathrm{N} \Delta \mathrm{T}$ and $\mathrm{M} \Delta \mathrm{T}$ are vectors that represent the in-plane force resultant, the moment resultant, the transverse shear force resultant, the thermal in-plane force resultant, and the thermal moment resultant, respectively. Also, $\mathrm{N} \Delta \mathrm{T}$ and $\mathrm{M} \Delta \mathrm{T}$ are given as:

$$
\left(\mathbf{N}_{\Delta \mathbf{T}}, \mathbf{M}_{\Delta \mathbf{T}}\right)=\int_{-h / 2}^{h / 2}(1, z) \mathbf{E}\{\alpha(T, z), \alpha(T, z), 0\}^{T} \Delta T(z) d z
$$

\subsection{Governing equations}

The principle of virtual work is used to derive the equations of motion, i.e.:

$$
\delta W=\delta W_{\text {int }}-\delta W_{\text {ext }}=0
$$

where $\delta W_{\text {int }}$ and $\delta W_{\text {ext }}$ represent the internal and external virtual work, respectively.

$$
\delta W_{\text {int }}=\int_{V} \delta \mathbf{e}^{\mathbf{T}} \boldsymbol{\sigma} d V=\int_{A}\left[\delta \boldsymbol{\varepsilon}^{\mathbf{T}} \mathbf{N}+\delta \mathbf{\kappa}^{\mathbf{T}} \mathbf{M}+\delta \boldsymbol{\gamma}^{\mathbf{T}} \mathbf{Q}\right] d A
$$




$$
\begin{aligned}
\delta W_{\mathrm{ext}}=-\int_{A} & {\left[I_{0}(u+v+\delta w)\right.} \\
& +I_{1}\left(\phi_{x}+\delta u+\phi_{y}+\delta v\right) \\
& \left.+I_{2}\left(\delta \phi_{x}+\delta \phi_{y}\right)-P_{a i r} \delta w\right] d A
\end{aligned}
$$

Cheng and Mei [4] tabulated the aerodynamic analysis categories according to the range of Mach numbers. As a result, nonlinear analysis of the structural model in hypersonic range can be calculated by the third-order piston theory. Also, the virtual work integral involving the surface stress vector is evaluated using the thirdorder piston theory to develop the aerodynamic loads on the surface of the plate as follows:

$$
P_{a i r}(x, y, t)=-\frac{\rho_{a} V_{\infty}{ }^{2}}{M_{\infty}}\left\{\begin{array}{l}
\frac{1}{V_{\infty}} w_{t_{t}}+w,_{x} \\
+\frac{\gamma+1}{4} M_{\infty}\left(\frac{1}{V_{\infty}} w,_{t}+w,_{x}\right)^{2} \\
+\frac{\gamma+1}{12} M_{\infty}{ }^{2}\left(\frac{1}{V_{\infty}} w,_{t}+w,_{x}\right)^{3}
\end{array}\right\}
$$

where $V_{\infty}, M_{\infty}, \rho_{a}, D$ and $\gamma$ are the airflow speed, the Mach number, the air density, the flexural stiffness matrix and ratio of specific heats, respectively.

Then, the governing equation is obtained as:

$$
\left(\mathbf{K}-\mathbf{K}_{\Delta \mathrm{T}}+\mathbf{A}_{\mathrm{f}}+\mathbf{A}_{\mathrm{f} 1}+\mathbf{A}_{\mathrm{f} 2}+\frac{1}{2} \mathbf{N} \mathbf{1}_{\mathrm{s}}+\frac{1}{3} \mathbf{N} \mathbf{2}_{\mathrm{s}}\right) \mathbf{d}_{\mathrm{s}}=\mathbf{P}_{\Delta \mathrm{T}}
$$

To analyse the thermal post-buckling behaviors of the FGM panel in hypersonic airflow, Newton-Raphson iterative method is employed

\section{Results and Discussions}

Figure 2 illustrates the center deflections of the clamped FGM plate for various volume fractions in different types. The deformations of the clamped S-FGM plate are appeared same deflections at upward and downward direction. In P-FGM type, as volume fraction index $\mathrm{k}$ is increased, deflection of the plate is risen. It is due to the volume fraction index $\mathrm{k}$ is increased, the amount of metal is grown in P-FGM plate. However behaviors of the S-FGM plate didn't have large gap by changing exponential k. Since volume proportions of ceramic and metal are made up same amount of the S-FGM by different volume fraction exponential $\mathrm{k}$.

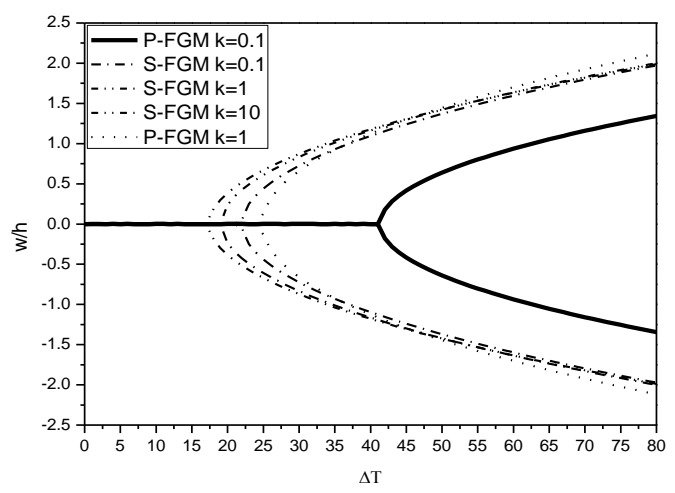

Fig. 2: Post-buckling of FGM models 


\section{Conclusions}

Thermal post-buckling behaviors of P-FGM, and S-FGM plates are investigated under aerodynamic pressure condition based on third-order piston theory. P-FGM plate has large gap of center deflection according to the volume fractions, while post-buckling deflection curves of the S-FGM plate are similar to various volume fraction index $k$.

\section{References}

[1] T. Hirano and T. Yamada, "Multi-paradigm expert system architecture based upon the inverse design concept," Int Work Art Intel Indust Appl, vol. 25, Hitachi, Japan, 1988.

https://doi.org/10.1109/AIIA.1988.13301

[2] J. N. Reddy, “Analysis of functionally graded plates,” Int J Num Meth Eng, vol 47, pp. 663, 2000. https://doi.org/10.1002/(SICI)1097-0207(20000110/30)47:1/3<663::AID-NME787>3.0.CO;2-8

[3] Y. L. Chung and S. H. Chi, "The residual stress of functionally graded materials," J Chinese Institute Civil Hydraulic Eng, vol. 13, pp. 1, 2001.

[4] G. Cheng and C. Mei, "Finite element modal formulation for hypersonic panel flutter analysis with thermal effects," AIAA J, vol. 42, pp. 687, 2004.

https://doi.org/10.2514/1.9553 